\title{
Precise Frost and Ice Detection for Defrost Scheme of Agricultural Refrigerator using Integrated Sensor Module based on Moving Window Method
}

\author{
Ji Hoon Seung ${ }^{1}$, Young Baik Kim², Dae Yeong Lim ${ }^{1}$ and Kil To Chong ${ }^{1}$, \\ ${ }^{1}$ Division of Electronic Engineering, Chonbuk National University, South Korea \\ ${ }^{2}$ KEPCO Engineering and Construction Company, Inc, South Korea \\ ${ }^{3}$ Advanced Electronics and Information Research Center, Chonbuk National \\ University, South Korea \\ *kitchong@jbnu.ac.kr
}

\begin{abstract}
In this paper, we present a method of measuring interruptions kinds of a frost and ice at radiator and control logic of agricultural refrigerator. Firstly, measurement of the frost and ice is important to reduce the operation efficiency of a refrigerator and prevent a malfunction. In order to precisely measure the interruptions factor, the fusion module using both the infrared sensor and pressure sensor is designed with moving window method. The performance of fusion module with adaptive moving window method is compared to designed low pass filter on steady state. The compared result is that adaptive moving window method is more suitable with agricultural refrigerator to measure interruption factors. Moreover, newly applied control logic shows the result of energy saving as compared with the conventional control method based on the measurement from designed sensor module set.
\end{abstract}

Keywords: refrigeration, infrared sensor, pressure sensor, control

\section{Introduction}

With the development of efficiency agricultural techniques combined with smart farming, the agricultural products are produced without season all over the world [1]. The produced products are exported to other places or countries in one of the vehicles, trains or ships for a long time or saved in the storages. To increase the values of products on moving or saving, it is needed to keep on fresh condition. Therefore, an operating scheme of the installed refrigerator at the transportation and warehouses is important to hold the product fresh.

There is, in addition, one further point to make. The energy conservation issues have been raised due to energy shortages caused by reduction of fossil fuels [2]. Refrigerators are used in various fields as following the previous reason. The elimination of the interruptions such as the frost and ice is a far-reaching challenge on keeping on fresh condition and the specific temperature from the energy conservation.

Since several years conventional refrigerators have been operated in a cyclic on-off manner for temperature regulation and certain period [3,4]. It leads to frequent start up and shutdown that affects the results on poor energy efficiency. Moreover, its scheme cannot control the refrigerator appropriately because it does not have a feedback loop system for the defrost.

In recent years, the various studies of the optimal control method have been researched by Luis Gomez Palacin [5], He Bingqiang [6] and Masato Kasahara [7]. However, these

Received (January 25, 2017), Review Result (May 11, 2017), Accepted (June 4, 2017)

* Corresponding Author 
studies focused on how well the refrigerator can keep on a goal temperature without an energy dissipation based on the system model.

In this paper, we deal with the defrost system with operating scheme of the agricultural refrigerator. In order to perform the detection of the interruptions, the module using both the infrared sensor and pressure sensor is designed and applied to the radiator. Specifically, the frost and ice is main factor in respect of decreasing the energy efficiency. The infrared sensor and pressure sensor, we used, have advantages of the ease to use and design circuits. In the other hand, the sensors have a lot of noisy of the measurement under the surrounding environment. Moving Window (MW) method is applied to eliminate the measurement noisy. MW method has been widely used in various fields such as robotics, mechatronics and sensor network. According to depend on the measurement from the designed sensor module, we can control the defrost system and plan to the operation for energy efficiency properly. Therefore, we can solve the problem of defrost and achieve the goal of energy saving.

The rest of this paper is organized as follows. The designed sensor module and MW method is in Section 2. Section 3 describes the control scheme of refrigerator system. The result of operating defrost system is given in Section 4. The conclusions are drawn in Section 5.

\section{Designed Sensor Module with Moving Window Method}

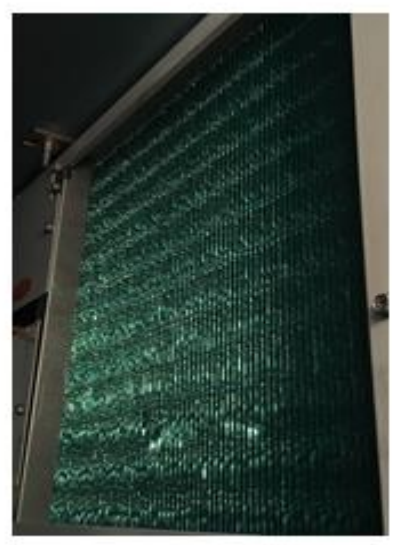

(a)

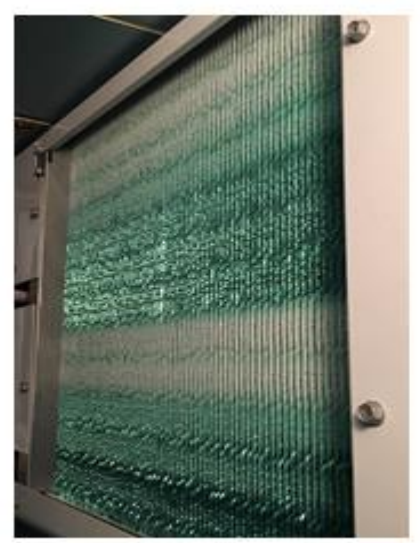

(b)



(c)

Figure 1. Frost and ice patterns in refrigerator; (a) steady state, (b) state after 2 hours, (c) state after 4 hours

\subsection{Integrated Sensor Design}

The designed sensor module is consisted of an infrared sensor and a pressure sensor with the advantages of low price, good consistency and easy to install.

In order to detect the frost and ice, firstly the patterns are shown in Figure 1. In Figure 1 , (a) is the steady state of refrigerator, (b) and (c) are the operating state after 2 hours and 4 hours, respectively.

In order to detect the interruptions, we have designed the sensor module described in Figure 2. The module has emitting diode, receiving transistor and pressure sensor.

When the interruptions exist on optical path, the receiving transistor does not receive the light from infrared emitting diode. There is also pressure sensor between the radiators to check the frost. 


\subsection{Moving Window Method}

MW method is used to eliminate the sensor noisy for increasing an accuracy of measurement.

There are many algorithms of the hardware types and software types, respectively.
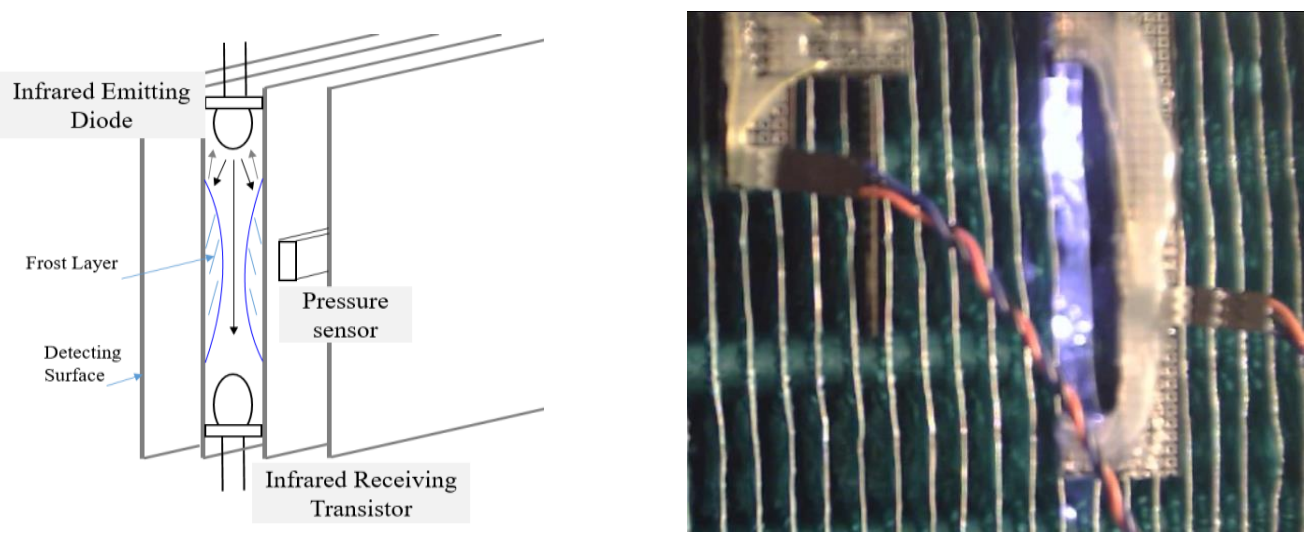

Figure 2. Optical Path Schematic of Designed Sensor Module and Test Bed

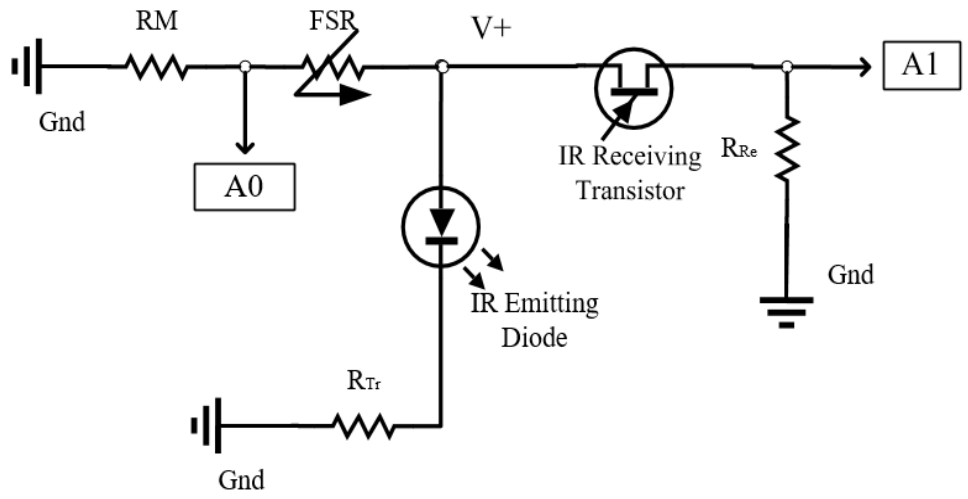

Figure 3. Circuit of Designed Sensor Module

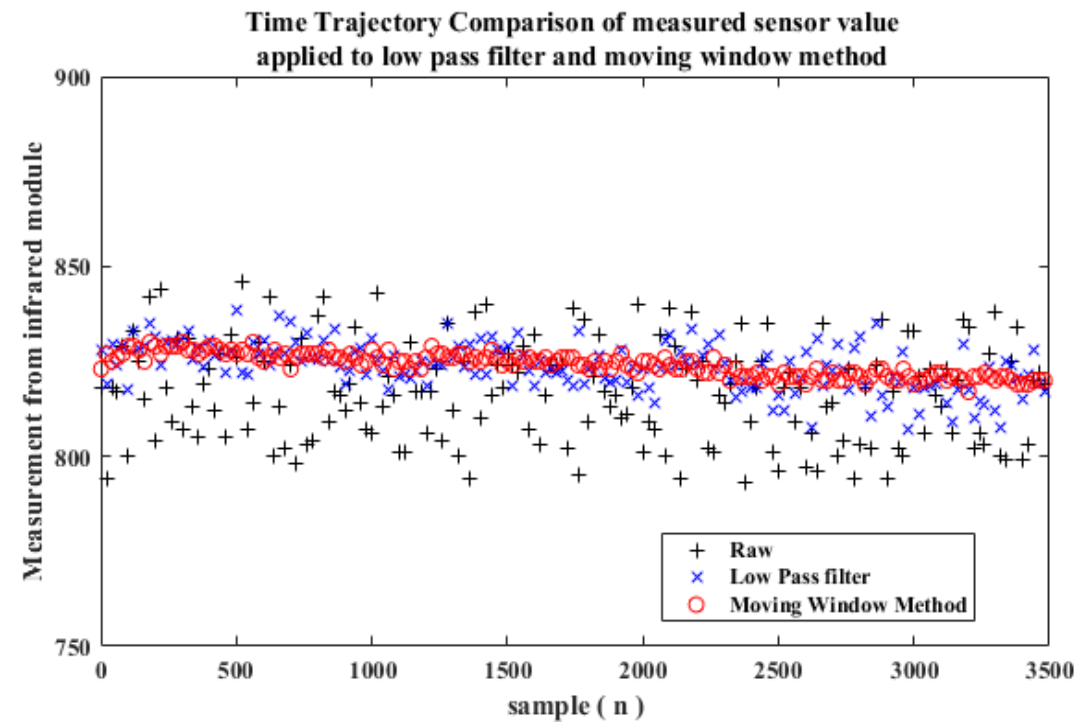

Figure 4. Time Trajectory Comparison of Raw Sensor Value, Values through Low Pass Filter and MW Method 
Table 1. Mathematical Analysis of Fig.2 Simple Test

\begin{tabular}{c|c|c|c}
\hline & $\begin{array}{c}\text { Raw measurement } \\
(1023 / 5 \mathrm{v})\end{array}$ & LPF & MW method \\
\hline Mean $(\bar{x})$ & 815.6886 & 817.9053 & 824.1874 \\
Standard deviation $(\sigma)$ & 67.8982 & 12.4073 & 2.9963 \\
\hline
\end{tabular}

Typical Operation of Heater System

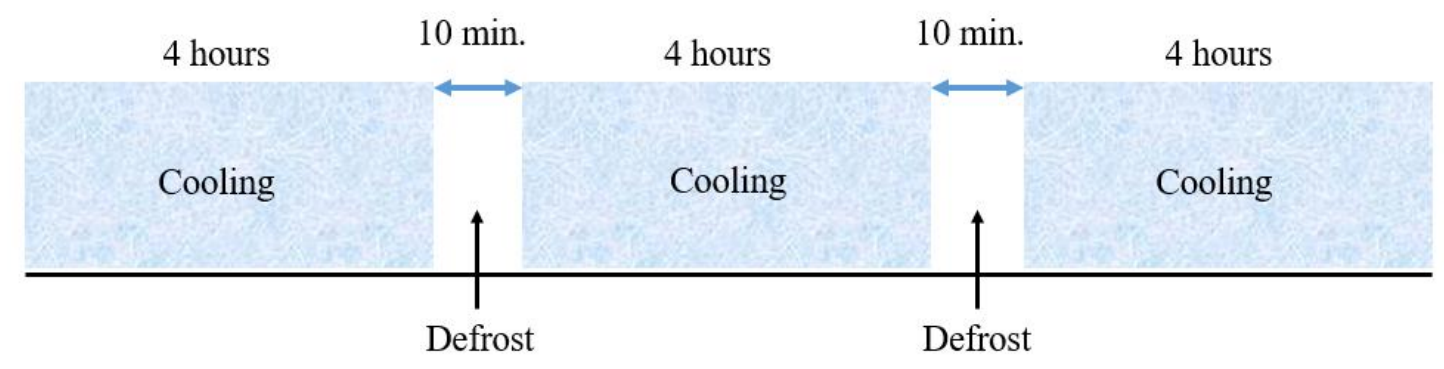

Figure 5. Example of Typical Operation of Cooling and Defrost System

We employ the software type for making the measurement data more stable.

An equations of MW method are as follows:

$$
y_{M W}=\frac{x_{n}+x_{n-1}+\cdots+x_{n-i}}{n},(i<n),
$$

where $y_{M W}$ is moving window measurement value, $x$ is a sensor value, $n$ and $i$ are denoted as window size and posterior set, respectively.

Figure 4 shows a simple example of infrared sensor and then the superiority of MW method that reduce the sensor noisy efficiently compared with the result of low pass filter.

In Figure 4, cross-dot line is raw infrared sensor value, $x$-dot line is the result of low pass filter and o-dot line represents the result of MW method. The data of simple test is collected for around 1 hours on stable states. The result of the simple test is summarized in Table. 1. MW method has better performances than others in the points of a mean and a standard deviation. Specifically, the MW method increases an accuracy about the quadruple comprised of LPF.

\section{Control Scheme of Defrost System}

Interruption factors are the frost and ice attached to the radiator grill but it is difference following the humidity and temperature of the air in a storage. Therefore, the Conventional defrost system have been operated in Fig. 5. For example, when the cooling is turned on for 4 hour, afterward, the mode of controller is switched as defrost system. It depends on the user setting. However, the user cannot control it appropriately for all that time.

In order to solve the problems, we used the designed sensor module to detect the frost and ice. The diagram and flow charts of refrigerator control scheme is shown in Fig. 6.

The data acquisition system can reach the Micro Control unit with obtained measurement. If the interruptions are detected, the defrost system is operated until the frost is eliminated ( it depends on user setting. ) 


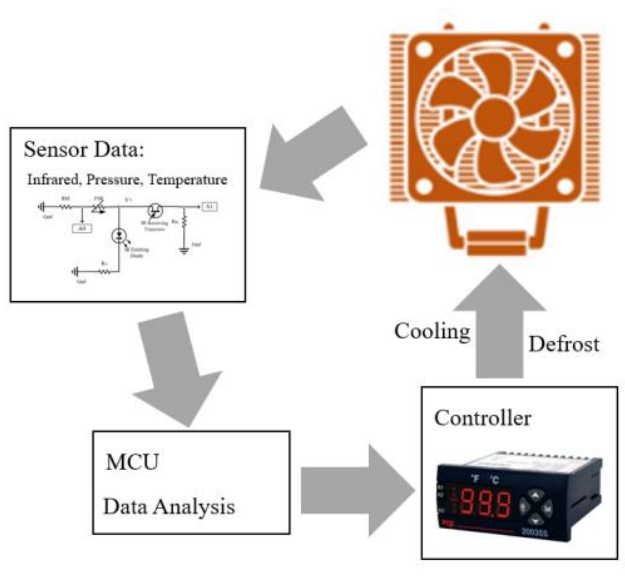

(a)

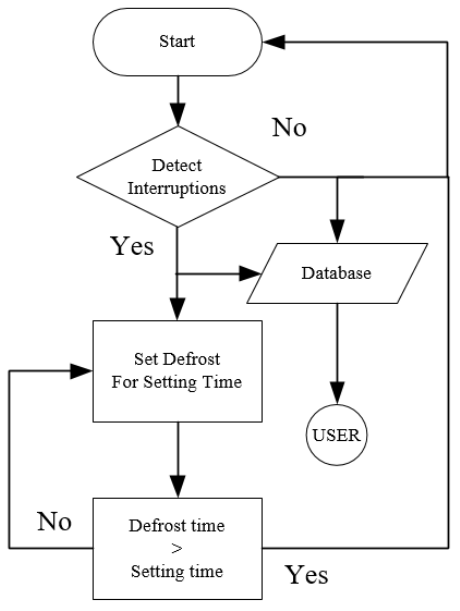

(b)

Figure 6. (a)Diagram of Flow Charts, (b) Defrost Control Scheme

The measurement system is consisted of the infrared sensor and pressure sensor. The data acquisition system including the control signal and data analysis is shown in Fig. 6 (a). The operating controller is FOX-2003SS made by Conotec. It allows an external signal to access defrost operating signal.

\section{Experiments and Results}

Test system in Figure 7 consists of the refrigerator in warehouse, main controller and data acquisition system with resistances, applied sensors (four infrared sensors set and two pressure sensors).



(a)

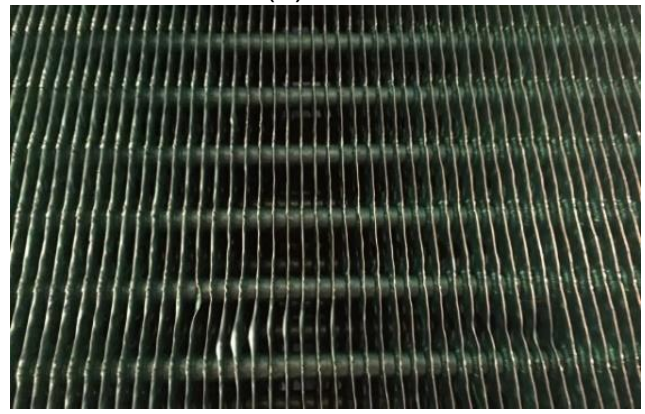

(c)



(b)

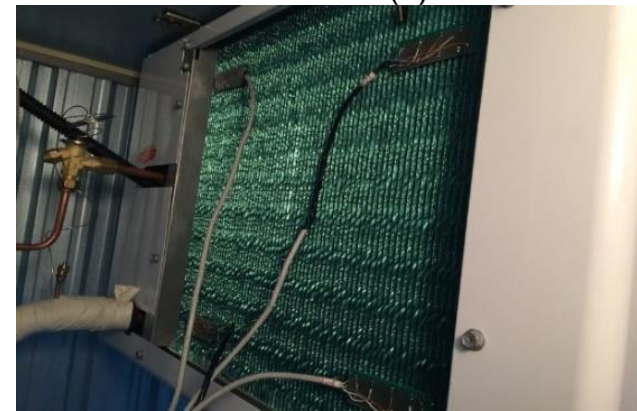

(d)

Figure 7. Test System: (a) Warehouse (b) Refrigerator (c) Radiator Grill (d) Sensor Set Consisted of Four Infrared Sensors and Two Pressure Sensors

In order to verify the performance of the adaptive defrost system, we experiment the cooling and defrost repeatedly. In Figure 7 (d), the integrated sensor modules are attached 
in each side of radiator grill in order to make the accuracy higher and checked.

The defrost control signal is based on the sensor module data to determine tuning on the refrigeration or defrost. When the sensor value is below 150 200, the data acquisition system determine that the interruptions occur. When the measurement of more three sensors is the value 1, than the defrost system is operated under the control schematic in Figure 6.

Figure 8 is a case 1 experiments captured from webcam and we can check that the interruption is grown as time passes without defrost operation. A case 2 set up the defrost system is operated for 10 minute after 4 hours shown in Figure 9. A case 3 using designed sensor module is set up and the defrost is determined by the acquisition system shown in Figure 10. The temperature of All cases is set up as $-20^{\circ} \mathrm{C}$ in same condition.



(a)

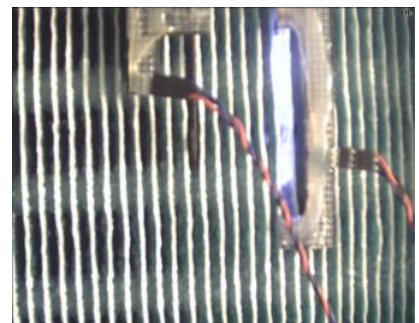

(b)

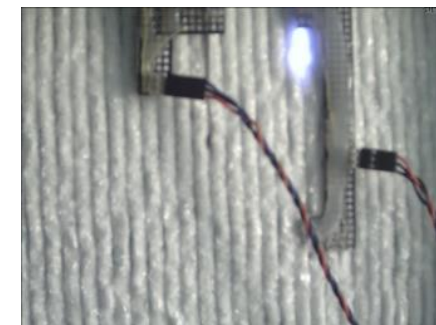

(c)

Figure 8. Results without defrost operation : (a) start (b) after 4 hours (c) after 8 hours

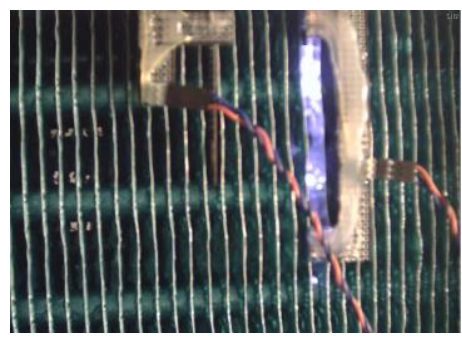

(a)



(b)



(c)

Figure 9. Results of case 2 : (a) start (b) after 4 hours (c) defrost operating



(a)

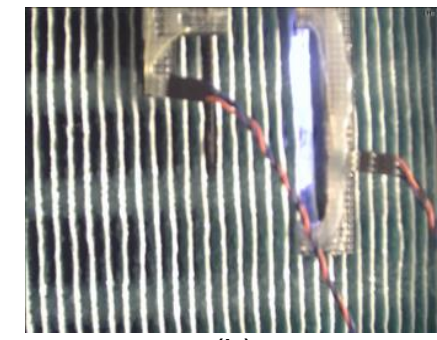

(b)

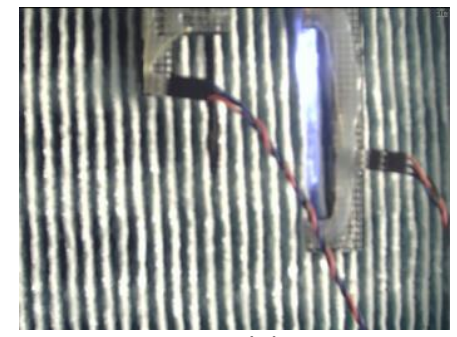

(c)

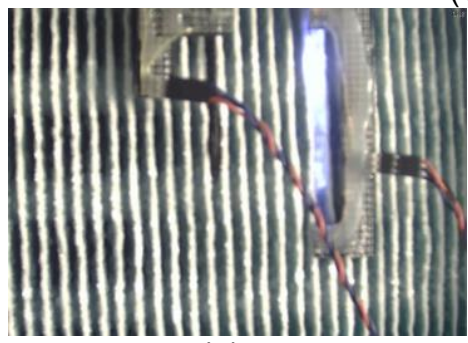

(d)



(e)

Figure 10. Results of Case 3 : (a) Start (b) after 4 hours (c) after 5 hours (d) Defrost Operating (e) Defrost is Finished 
We can check the importance of defrost system using the case 1 in Figure 8 . The interruptions have been imbued in the radiator grill of refrigerator after 8 hours from start. The case 2 shows the elimination of the interruptions after 4 hours. However, the refrigeration does not need to defrost operation in Figure 9 (b). When the defrost system is operated, the temperature is necessarily going up. After finishing defrost work, the refrigerator has to operate more time to compensate temperature loss and match the goal temperature. The case 3 using designed sensor module shows that the interruption is eliminated well properly. Moreover, this case keep the cooling time without going up the temperature compared with case 2.

The time trajectory of interruption measurement and defrost operation is shown in Figure 11 .

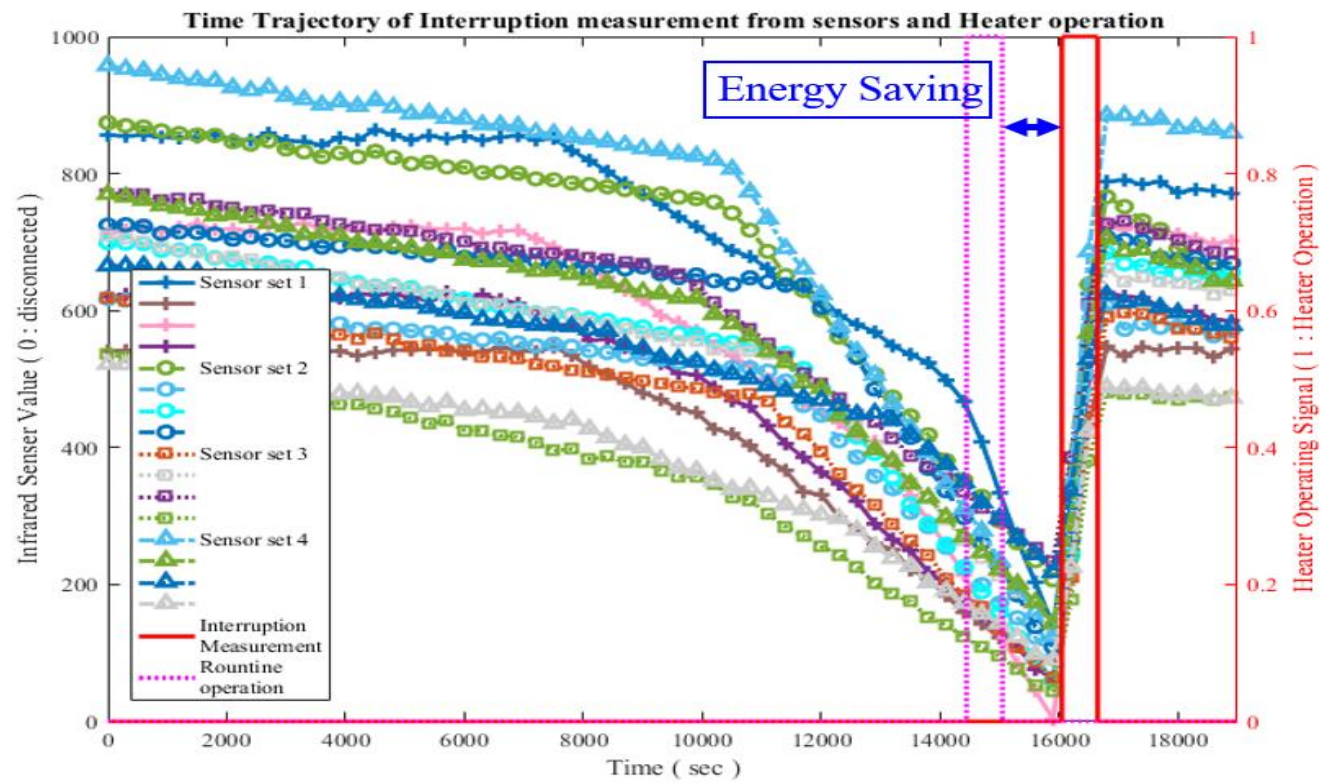

\section{Figure 11. Time Trajectory of Interruption Measurement and Defrost Operation}

\section{Conclusion}

Generally, the refrigeration operates a routine in 4 times or 5 times in a day. However, its scheme is not efficiency because it cannot be adjustable in each situation of refrigeration such as how much the ice or frost is in the radiator and more than expected ice or frost occur as effecting external environments or the door of refrigeration is open. Therefore, we present a method of measuring a frost and ice to increase an efficiency of refrigeration, also the infrared sensor and pressure sensor are applied to obtain an accurate data. The results of experiments show that the interruption can be obtained well and save the energy more efficiently than typical routine operation. The further plan is calculating how much the energy can be saved using the inverter control.

\section{Acknowledgments}

This research was financially supported by the Ministry of Trade, Industry and Energy(MOTIE), Korea Institute for Advancement of Technology(KIAT) through the International Cooperative R\&D program. (N046200012) and the Brain Korea 21 PLUS Project and Business for Cooperative R\&D between Industry, Academy, and Research Institute funded Korea Small and Medium Business Administration in 2016 (Grants No.1601001522). 


\section{References}

[1] D. S Jung, R Radermacher "Performance simulation of a two-evaporator refrigerator-freezer charged with pure and mixed refrigerants", Journal of Refrigeration, Vol. 14, pp. 254-263, (1991)

[2] Emiliano Schena, Daniele Tosi, Paola Saccomandi, Elfed Lewis, Taesung Kim, "Fiber Optic Sensors for Temperature Monitoring during Thermal Treatments: An Overview", Sensors, 16 (2016)

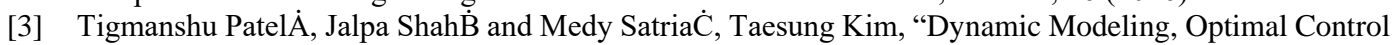
Design and Comparison between various control schemes of Home Refrigerator", International Journal of Current Engineering and Technology, (2013) 2047-2052

[4] Chang-Duk Jeon, Dong-Seon Kim, Seung-Jun Lee, "Feasibility Study on a Defrost Control Method by Using a Photoelectric Sensors", Journal of the Korea Academia-Industrial cooperation Society, Vol. 15, No. 6 (2014), 3389-3395

[5] Luis Gomez Palacina, Benjamin Bradua, Enrique Blanco Vinuelaa, Ryuji Maekawab, Michel Chalifourb, "An optimal control approach for an overall cryogenic plant under pulsed heat loads", 25th International Cryogenic Engineering Conference and the International Cryogenic Materials Conference (2014),

[6] He Bingqiang, Liao Chunling, "The Research on Optimal Control of Hvac Refrigeration System", IJCSI International Journal of Computer Science Issues, Vol. 9, Issue 5, No 2, September (2012), 65-70

[7] Masato Kasahara, Tadahiko Matsuba, Yukihiro Hashimoto, Itaru Murasawa, "Optimal preview control for HVAC system", ASHRAE Transactions, Vol 104 (1998). 4145-4156 